\title{
Conditions and means for a useful and trustworthy engagement of experts in the PREPARE analytical platform - survey and interviews outcomes
}

\author{
M. Montero ${ }^{1}$, R. Sala ${ }^{1}$, C. Trueba ${ }^{1}$ and S. Baudé ${ }^{2}$ \\ ${ }^{1}$ CIEMAT - Environment Department, Avenida Complutense 40, 28040 Madrid, Spain. \\ ${ }^{2}$ MUTADIS - 5 rue d'Alsace, 75010 Paris, France.
}

\begin{abstract}
The PREPARE analytical platform (AP) has been developed under the framework of the European project PREPARE (PREPARE. "Innovative integrated tools and platforms for radiological emergency preparedness and postaccident response in Europe", EC FP7-EURATOM-FISSION GA 323287. http://www.prepare-eu.org) to be a focal point for collecting information, analysing any nuclear or radiological event and providing information about the consequences and its future evolution. In this paper, research to look for and involve the potential users of the AP was accomplished. Specifically, the study aimed to evaluate, first, the perceptions and expectations of potential users about usefulness, trustworthiness, interaction with other users and governance offered by the AP, and second, the willingness and interest of potential users to join the platform and contribute with their expertise. The final goal was to identify the conditions and means for a useful and trustworthy engagement of experts in the AP. The two approaches used to reach this goal are described here, based on an on-line questionnaire and personal interviews to institutional and noninstitutional experts, authorities and international organisations. The main findings and conclusions obtained are presented.
\end{abstract}

Keywords: analytical platform / information management / nuclear crisis / engagement of expert / survey

\section{Introduction}

The analytical platform (AP) has been developed under the PREPARE project with the general aim to provide an easy access platform for information exchange in time of a nuclear or radiological crisis (Raskob et al., 2016). Being a web-based application, it is necessary to look for and involve the potential users of the PREPARE AP. For this purpose, the task group dedicated to the "Operational Procedures of the AP" (WP2.2), in collaboration with the task group WP6.4 on "Information and participation of the public-coordination \& integration", have accomplished a survey to specifically evaluate: (i) the perceptions and expectations of potential users about the usefulness, trustworthiness and interaction with others users and governance offered by the AP; and (ii) the willingness and interest of potential users to join the AP and contribute with their expertise.

To help the establishment of these objectives, two approaches have been used:

- an on-line questionnaire (OQU), addressed to the NERIS ${ }^{1}$

Platform community and partners in the PREPARE

\footnotetext{
${ }^{1}$ NERIS. "European Platform on preparedness for nuclear and radiological emergency response and recovery". http://www.eu-neris. net/.
}

project, managed by CIEMAT from WP2.2 (Montero et al., 2016);

- personal interviews (INT) among institutional and noninstitutional experts, authorities and international organisations, realised by Mutadis from WP6 (Baudé et al., 2016).

The methodology used in each approach, the summary of the findings and the main conclusions obtained in the study are presented in the following sections.

\section{Methodology}

Regarding the OQU, CIEMAT ${ }^{2}$ has been in charge of its design, in collaboration with $\mathrm{KIT}^{3}{ }^{3} \mathrm{VUJE}^{4}$ and $\mathrm{APA},{ }^{5}$ managing and analysing the answers received. NERIS community and partners of PREPARE (73 organisations altogether) were asked to participate in the survey. A total

\footnotetext{
${ }^{2}$ CIEMAT - Centro de Investigaciones Energéticas, Medioambientales y Tecnológicas (Spain). http://www.ciemat.es/.

${ }^{3}$ KIT - Karlsruhe Institute of Technology (Germany). https://www. kit.edu/english/.

${ }^{4}$ VUJE - VUJE, a.s. (Slovak Republic). www.vuje.sk/.

${ }^{5}$ APA - Portuguese Environment Agency (Portugal). http://www. apambiente.pt/index.php.
} 
of 34 responses were received with a response rate around $47 \%$.

The final version of the OQU was composed of 19 questions grouped in seven subjects or categories of interest: 'Information and knowledge', 'Usefulness', 'Trustworthiness', 'Expectations', 'Interactions and information sharing', 'Interest in joining the AP' and 'Governance'. Each category had questions following different requirements: (i) rated on a scale of 1-5 based on the grade of agreement with the statement of the question; (ii) a single-select multiple choices or (iii) ranking the items. In addition, questions with openended text complemented these answers. From the replies obtained, the importance of the different statements proposed was evaluated, and the main findings regarding each subject was identified.

The INT had 13 questions open-ended, grouped in six categories: 'Level of information and awareness', 'Usefulness of the platform', 'Interest in joining the platform', 'Conditions for safe and reliable engagement into the platform', 'Conditions and means for trustworthy interactions between experts' and 'Management \& governance'. A total of 12 interviews, including PREPARE members, institutional experts, regulators, NGO experts and international institutions (OECD-NEA, ${ }^{6}$ ETSON $^{7}$ HERCA $^{8}$ ), were obtained from 15 requests.

The main findings from the joint qualitative assessment of the results are shown as follows, grouped in six general topics.

\section{Summary of?the main findings}

\subsection{Information and knowledge}

Regarding this topic, in general the two approaches show the same trend. The results from the OCQ show that most of the NERIS Platform community and Partners in the PREPARE project were moderately informed on the AP (59\% of replays from OQU). Most of interviewees outside this community were unaware of its existence. This misinformation could favour that the AP is perceived as duplicate of other tools sharing information.

\subsection{Usefulness of?the platform}

It looked like that the AP is more useful for experts $(50 \%$ respondents from OQU) and not for the public (12\%). The INT revealed that the AP is more likely to be used by experts in the case of a more distant accident, or after the emergency phase. In both studies, the AP was recognised as very valuable to centralize the information, enhancing the collaboration and exchange between experts. It was stressed that the AP should not duplicate existing tools for international information sharing (e.g. the ECURIE European tool).

\footnotetext{
${ }^{6}$ OECD-NEA - Nuclear Energy Agency under the Organisation for Economic Co-operation and Development. https://www.oecd-nea. org/.

${ }^{7}$ ETSON - The European Technical Safety Organisations Network. http://www.etson.eu/Pages/Homepage.aspx.

${ }^{8}$ HERCA - Heads of the European Radilogical Protection Competent Authorities. http://www.herca.org/index.asp.
}

\subsection{Trustworthiness}

The general perception of the OQU participants was that the $\mathrm{AP}$ is a more trustworthy tool for experts (47\%) than for the general public (29\%), based on items such as experts' participation; timely and reliable information and openness in discussion, including uncertainties. The respondents from INT highlighted the need of establishing clear terms of reference, the operation rules, the code of conduct and governance rules.

\subsection{Expectations, interactions and information sharing}

The AP was expected, based on relies from the OQU, as a useful tool to collect and share information, exchange experience and interact with others in an open, easy and quick manner, stressing its building on internationally accepted operational structures. Regarding experts' interactions, the main debate from OQU was the use of conventional versus technological tools, with a clear tendency for the second option. Regarding the interactions with the public, the information should be understandable and clear including and explaining uncertainties. The respondents of the OQU declared to be willing $(47 \%)$ or very willing $(28 \%)$ to share information with the public.

The interviewees, on the other hand, were focussed on the type of information to be shared, demanding identification of the experts taking part in the AP, confidentiality and explicit rules for the operation. In the case of interaction with the public, it was highlighted that consensus and dissent should be made explicit with transparent and validated information.

\subsection{Interest and relevant issues for joining the platform}

Altogether, the interest in joining the AP relies primarily in meeting the expectations and wishes expressed previously, namely: (i) possibility of increasing knowledge, and easy, quick and reliable information sharing, (ii) opportunity to interact with other stakeholders, having access to simulation tools as well as to help and interact with others scientists. Most of the participants in OQU $(78 \%)$ reported to be interested in joining the AP, due to "the use of the outcomes and the conditions for information dissemination". The INT replied on this topic mainly stressing that the engagement with the AP will depend on the range of actors involved and on the level of political support from governments, authorities and expert institutions.

\subsection{Governance}

Two questions were addressed: Who should lead the AP and how decisions should be taken. Regarding the first question, the NERIS Platform would be the preferred body for the leadership of the platform (with $72 \%$ of the replies), followed by an international organisation (66\%) and a national or private organisation $(56 \%)$ in the third place. The preferred options to take decisions, were by consensus and/or by (operational) management board, followed by an operational/ chief executive officer. 


\section{Conclusions}

The two approaches followed seem valid for the purposes of the research with an acceptable response rate of $(47 \%$ and $80 \%$ for OQU and INT, respectively). The target of the first one has been larger than the second one and the type of participants slightly different. Although the persons directly related to the issue of EP\&R are informed or well informed on $\mathrm{AP}$, it is necessary to expand the dissemination of information beyond this collective to avoid misinformation on the purpose and possibilities of the AP in relation to other existing tools for sharing information.

It was stressed that the AP would be a more useful and trustworthy tool for experts than for the general public. Notwithstanding, the experts also highlighted the importance of transparency in communication declaring their willingness to share information with the public. Joining the AP would be conditioned to the fulfilment of the declared expectations: useful tool to collect and share information, exchange experience and interact with others in an open, easy and quickly manner, avoiding duplication efforts when making decisions. Indeed, the support of the tool would be favoured by existing operational structures internationally accepted to have continuous testing and by establishing clear terms of reference, operation rules and codes of conduct addressed to the best governance. The NERIS Platform would be the preferred body for the leadership of the platform, and the decisions would be taken by an (operational) management board or consensus.

Acknowledgement. The research leading to these results has received funding from the European Atomic Energy Community Seventh Framework Programme FP7/2012-?2013 under grant agreement 323287.

\section{References}

Baudé S., Hériard-Dubreuil G., Marignac Y., Hazemann J., Perko T., Eikelmann I.M. (2016) Final transversal framework including lessons, principles and guidelines on information and participation in nuclear emergency and post-emergency (CE-FP7-fission-2012, PREPARE GA No 323287). Technical Deliverable D6.19.

Montero C., Sala R., Trueba C. (2016) Conditions and means of reliable and trustworthy engagement in the PREPARE analytical platform. Outcomes from an on-line survey addressed to the EP\&R Community (CE-FP7-fission-2012, PREPARE GA No 323287). Technical Deliverable D2.15, PREPARE(WP2)-16-01.

Raskob W. et al. (2016) Overview and applicability of the analytical platform, Radioprotection 51 (HS2), S179-S180.

Cite this article as: M. Montero, R. Sala, C. Trueba, S. Baudé. Conditions and means for a useful and trustworthy engagement of experts in the PREPARE analytical platform - survey and interviews outcomes. Radioprotection 51(HS2), S195-S197 (2016). 